\title{
Development of High Pressure Freezing and Correlative Light/Electron Microscopy for Drosophila Larvae
}

\author{
Linda S. Nikolova ${ }^{1,2}$ and Mark M. Metzstein ${ }^{2}$
}

${ }^{1}$ Electron Microscopy Core Laboratory and ${ }^{2}$ Department of Human Genetics, University of Utah, Salt Lake City, Utah, USA

We are interested in the mechanisms by which cells generate and maintain their complex architectures. A major structural element in cells are lipid membranes, which define both the external cell shape as well as forming subcellular compartments, such as organelles. Transmission electron microscopy (TEM) remains a method of choice for examine cell ultrastructure, particularly for examining cellular membranes. Fixation methods that maintain membranes in their native states are thus critical for examining cells using TEM. One such method is high pressure freezing (HPF), in which rapid freezing under high pressures is used to prevent the formation of water ice crystals that otherwise disrupt cellular architecture. ${ }^{1}$

We study cellular architecture in the fruit fly Drosophila melanogaster. By using Drosophila, we can take advantage of the numerous genetic and molecular tools available in this organism. For instance, we can isolate mutants that have defects in cell development, thus identifying genes critical for formation of proper cell shape. We focus on a specific cell type of the Drosophila respiratory system called terminal cells. In larval stages, terminal cells form dozens of fine subcellular branches (Fig. 1). Each of these branches also contains a membrane-bound intracellular lumen, through which oxygen and other gases flow (Fig. 1). To examine the ultrastructure of terminal cells, we have adapted the HPF technique to fix larval Drosophila. We have developed a method that leads to excellent preservation of cellular membranes (Fig. 2A), including both the plasma membrane as well as intracellular organelles. We are now using this method to characterize terminal cell architecture (Fig. 2B) and, for the first time, identified a candidate membrane intermediate in the formation of the subcellular lumen (Fig. 2C).

One particular advantage of using Drosophila, is we can generate transgenic animals in which a tissue of interest expresses a fluorescent protein marker, such as GFP or DsRed. Using HPF, we have developed methods to fix larvae in such a way that fluorescence of these transgenic markers is maintained. This so called correlative light /electron microscopy (CLEM) procedure ${ }^{2,3}$ allows us to identify cells using light microscopy and then perform TEM on the same samples (Fig. 3). We are now using our technique to characterize Drosophila mutants in which lumen formation fails in terminal cells. Ordinarily, such cells are very hard to identify by TEM, since the presence of the lumen is the defining characteristic of a terminal cell branch. However, we can now use our CLEM procedure to identify mutant cells in sections based on their expression of a cell-type specific fluorescent marker, and subsequently characterize the cell ultrastructure using TEM.

1. McDonald, K. L. and Auer, M. Biotechniques 41 (2006), p137.

2. Brown, E., et al. Semin Cell Dev Biol 20 (2009), p910.

3. McDonald, K. L.. J Microsc 235 (2009), p273. 


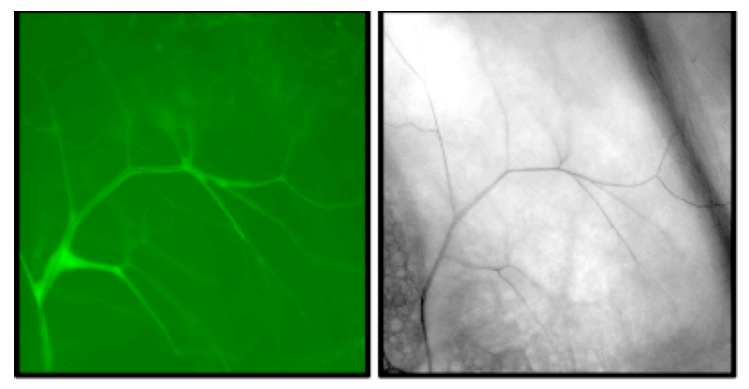

Figure 1. Left, a single wild-type tracheal terminal cell in a Drosophila larva, labeled with GFP. Right, each branch contains an air-filled lumen, which can be observed in the bright-field view due to the refractive index difference with the surrounding fluid-filled tissue.
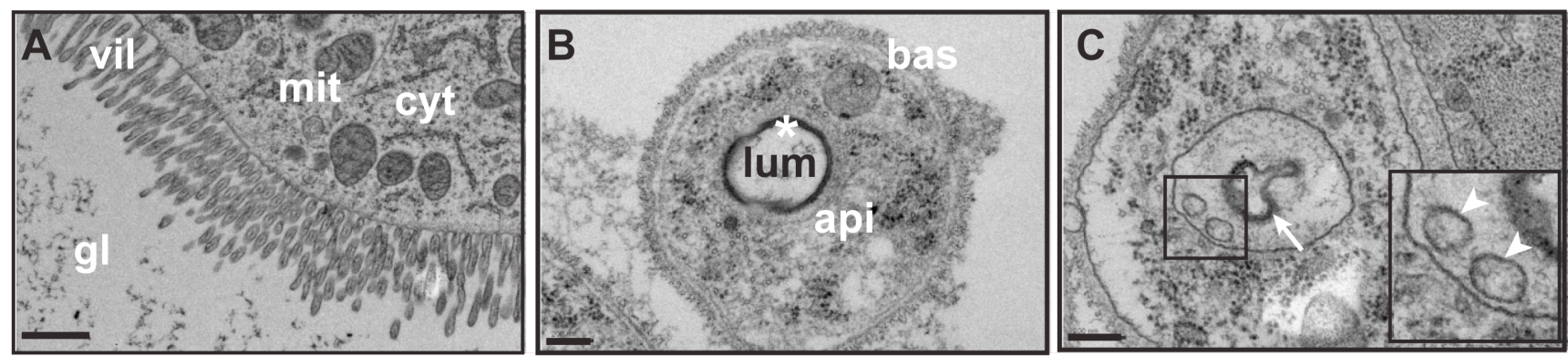

Figure 2. A Example of a Drosophila larva fixed using HPF. Microvilli (vil) on the gut apical surface and mitochondria (mit) within the cell cytoplasm (cyt) are clearly visible. gl = gut lumen. B Cross section of a tracheal terminal cell branch showing the branch basal cell membrane and lamina (bas) and apical membrane (api) surrounding the lumen (lum). The lumen is lined with an elaborate chitinous matrix (asterisk). C Candidate immature luminal structure shows a multi-membrane intracellular compartment with vesicles (arrowheads in inset) with a limiting membrane and a maturing luminal chitin matrix (arrow). Scale bars $=200 \mathrm{~nm}$.
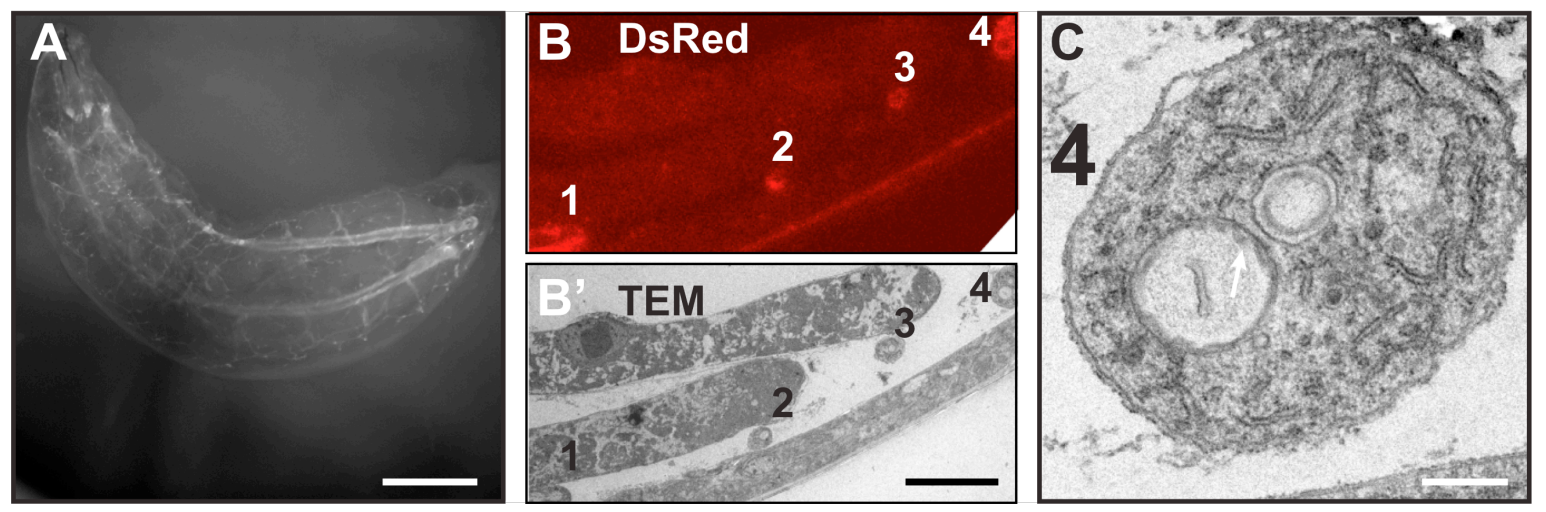

Figure 3. A Whole-mount of a Drosophila larva expressing DsRed fluorescent throughout the tracheal system, fixed by HPF and embedded for TEM sectioning. B Section of larvae showing DsRed expression in tracheal branch profiles (labeled 1-4) and $\mathbf{B}^{\prime}$ adjacent section imaged by TEM. C Close up of tracheal branch profile \#4. Scale bars: $0.5 \mathrm{~mm}(\mathrm{~A}) ; 5 \mu \mathrm{m}\left(\mathrm{B}, \mathrm{B}^{\prime}\right) ; 500 \mathrm{~nm}(\mathrm{C})$ 